ARTICLE

Received 7 Jun 2014 | Accepted 11 Feb 2015 | Published 18 Mar 2015 DOl: 10.1038/ncomms7620

\title{
Single-step deposition of high-mobility graphene at reduced temperatures
}

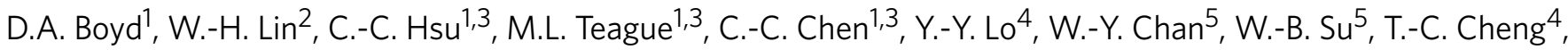 \\ C.-S. Chang 5 , C.-I. $\mathrm{Wu}^{4} \&$ N.-C. Yeh ${ }^{1,3,6}$
}

Current methods of chemical vapour deposition (CVD) of graphene on copper are complicated by multiple processing steps and by high temperatures required in both preparing the copper and inducing subsequent film growth. Here we demonstrate a plasma-enhanced CVD chemistry that enables the entire process to take place in a single step, at reduced temperatures $\left(<420^{\circ} \mathrm{C}\right)$, and in a matter of minutes. Growth on copper foils is found to nucleate from arrays of well-aligned domains, and the ensuing films possess sub-nanometre smoothness, excellent crystalline quality, low strain, few defects and roomtemperature electrical mobility up to $(6.0 \pm 1.0) \times 10^{4} \mathrm{~cm}^{2} \mathrm{~V}^{-1} \mathrm{~s}^{-1}$, better than that of large, single-crystalline graphene derived from thermal CVD growth. These results indicate that elevated temperatures and crystalline substrates are not necessary for synthesizing high-quality graphene.

\footnotetext{
${ }^{1}$ Department of Physics, California Institute of Technology, Pasadena, California 91125, USA. ${ }^{2}$ Department of Applied Physics, California Institute of Technology, Pasadena, California 91125, USA. ${ }^{3}$ Institute of Quantum Information and Matter, California Institute of Technology, Pasadena, California 91125, USA. ${ }^{4}$ Graduate Institute of Photonics and Optoelectronics, Department of Electrical Engineering, National Taiwan University, Taipei, Taiwan 10617. ${ }^{5}$ Institute of Physics, Academia Sinica, Nankang, Taipei, Taiwan 115. ${ }^{6}$ Kavli Nanoscience Institute, California Institute of Technology, Pasadena, California, 91125 , USA. Correspondence and requests for materials should be addressed to N.-C.Y. (email: ncyeh@caltech.edu).
} 
$\mathrm{M}$ uch progress has been made in growing large-area graphene by means of thermal chemical vapour deposition (CVD) based on catalytic dehydrogenation of carbon precursors on copper ${ }^{1-4}$. However, in many instances it is desirable to avoid multiple steps ${ }^{2}$ and high temperatures $\left(\sim 1,000^{\circ} \mathrm{C}\right)$ employed in thermal-CVD growth. In particular, the conditions for the critical removal of the native copper oxide and the subsequent film growth are dissimilar enough to necessitate separate process steps. Moreover, high processing temperatures restrict the types of devices and processes where CVD can be applied and can also result in film irregularities that compromise the graphene quality itself ${ }^{4}$. Thermally derived strain and topological defects, for example, can induce giant pseudo-magnetic fields and charging effects ${ }^{5-8}$, giving rise to localization and scattering of Dirac fermions ${ }^{7,8}$ and diminishing the electrical properties.

A variant of thermal CVD, called plasma-enhanced CVD (PECVD), has been widely used for depositing many allotropes of carbon, most notably diamond films. The plasma can provide a rich chemical environment, including a mixture of radicals, molecules and ions from a simple hydrogen-hydrocarbon feedstock ${ }^{9}$, allowing for lower deposition temperatures ${ }^{10}$ and faster growth than thermal CVD. The ability of the plasma to support multiple reactive species concurrently is a key advantage. However, the quality of PECVD-grown graphene to date has not been significantly better than that of thermal $\mathrm{CVD}^{10-12}$.

Here we demonstrate a PECVD process that encompasses both the preparation of the copper and growth of graphene in a single step. The addition of cyano radicals, which are known to etch copper at room temperature ${ }^{13}$, to a hydrogen-methane plasma is found to produce a chemistry whereby the native oxide is removed, the copper is smoothly etched, and growth of wellaligned graphene ensues. Etching of the copper substrate is found to be self-limiting, allowing etching and growth steps to proceed in tandem under the same chemistry. The entire process occurs in a matter of minutes without active heating, and the resulting graphene films exhibit high electrical mobility and few structural defects. Our results indicate that elevated temperatures, crystalline growth substrates, and long process times are not necessary for synthesizing high-quality graphene.

\section{Results}

Description and characterization of process. The PECVD growth process is schematically illustrated in Fig. $1 \mathrm{a}-\mathrm{c}$ and further described in Methods and Supplementary Fig. 1. Copper was directly exposed to a low-pressure, microwave hydrogen plasma containing trace amounts of cyano radicals, methane and nitrogen (Supplementary Fig. 2 and Supplementary Fig. 3), as detailed in Supplementary Note 1. Removal of the native oxide and smoothing of the copper typically occurred within $2 \mathrm{~min}$ of igniting the plasma (Fig. 1a). Nucleation of graphene ensued on both sides of the substrate (Fig. 1b). With continued exposure to the plasma, disordered graphite and monolayer graphene covered the top and bottom sides, respectively (Fig. 1c). Copper deposits were found on both the process tube and the sample holder after successful growth runs (Fig. 1d), whereas runs with little copper etching did not produce optimal films. Optical microscopy showed that the top surface of the sample was always randomly pitted after growth (Fig. 1e), whereas that on the bottom surface was smooth (Fig. 1f). Scanning tunnelling microscopy (STM) measurements of the surface topography of graphene on the bottom side of $\mathrm{Cu}$ revealed sub-nanometre flatness for samples grown on $\mathrm{Cu}$ foils and single crystalline $\mathrm{Cu}$ (100) and $\mathrm{Cu}$ (111) (Fig. 1g-i and Supplementary Fig. 5a-c). Nitrogen incorporation into the graphene, as measured by X-ray photoemission spectroscopy, Supplementary Fig. 4a,b, was found to be below the detection limit ${ }^{14}$ of 0.1 at. $\%$.

We found that the occurrence of PECVD-graphene growth was largely insensitive to the gas temperature of the plasma. The maximum gas temperature of the plasma determined by a thermocouple sheathed in boron nitride was found to be $160^{\circ} \mathrm{C}$ $\left(425^{\circ} \mathrm{C}\right)$ for $10 \mathrm{~W}(40 \mathrm{~W})$ plasma power, which decayed by more than $120^{\circ} \mathrm{C}\left(250^{\circ} \mathrm{C}\right)$ within $\sim 1 \mathrm{~cm}$ from the centre of the plasma. The maximum temperature of the copper substrate $T_{\mathrm{s}}$ was measured using the melting points of known solids on top of a copper substrate subjected to the plasma. We found that at $40 \mathrm{~W}$ plasma power, lead melted whereas zinc did not, indicating that the maximum $\mathrm{Cu}$ substrate temperature was within the range of $327.5^{\circ} \mathrm{C}<T_{\mathrm{s}}<419.5^{\circ} \mathrm{C}$. Further, we were able to fabricate more than 300 high-quality large-area graphene samples by PECVD within 5-20 min in a single-step by using plasma power varying between 10 and $40 \mathrm{~W}$. The typical substrate size was $(8 \times 13) \mathrm{mm}^{2}$. Although the plasma produced by the Evenson cavity was not uniform in intensity over the length of the sample, graphene was found to cover the entire substrate and exhibited consistent quality over the entire film on the bottom side, indicating that the growth was not temperature sensitive and could occur over a range of temperatures. We expect that the sample size should be scalable in a larger cavity under the same growth conditions. The localized nature of the plasma source also allowed multiple samples to be prepared individually in the same process tube without changing conditions or breaking vacuum by simply translating the Evenson cavity.

Nucleation and growth. The time evolution in the Raman spectra of PECVD-graphene on copper foils during growth is shown in Fig. 1j,k. Spectra from the top side (Fig. 1j) featured the D-band, an indicator of edges or disorder in graphene and graphite; the G-band, a feature common to both graphene and graphite; and the 2D-band, a feature that can be used to distinguish graphene from graphite $^{15}$. The D-band was prominent throughout, whereas the 2D-band decreased and disappeared with time, indicating the formation of disordered graphite, which is consistent with optical images of the top side (Fig. 1e). In contrast, spectra from the bottom side (Fig. 1k) showed that the D-band decreased and eventually vanished with increasing growth time, whereas the relative intensities of $2 \mathrm{D}$ and G-bands indicate the formation of monolayer graphene ${ }^{15-18}$, as described in Methods and Supplementary Fig. 6a,b. This behaviour is consistent with growth and eventual coalescence of graphene domains wherein the D-band associated with the edge states of the domains diminished $^{19}$. A comparison of the graphene from the top and bottom sides indicate that increased mass flux and direct plasma exposure adversely affected the film quality, and hereafter we focus our studies on PECVD-graphene grown on the bottom side of $\mathrm{Cu}$.

Scanning electron microscopy (SEM) images taken shortly after the onset of graphene growth on copper foil (Fig. 2a-d) revealed dense, linear arrays of hexagonal graphene domains that extended across the copper grains. With increasing growth time, the wellaligned graphene domains coalesced seamlessly into a monolayer graphene with few defects. Shown in Fig. 2e,f are the 2D/G and $\mathrm{D} / \mathrm{G}$ intensity ratio maps of a $(100 \times 100) \mu \mathrm{m}^{2}$ area of an as-deposited graphene sample on $\mathrm{Cu}$, respectively. The average $2 \mathrm{D} / \mathrm{G}$ intensity ratio $\left(I_{2 \mathrm{D}} / I_{\mathrm{G}}\right)$ is $\sim 2$ and only $5 \%$ of the mapped area show any measurable D-band. Histograms of the $\left(I_{2 \mathrm{D}} / I_{\mathrm{G}}\right)$ and $\left(I_{\mathrm{D}} / I_{\mathrm{G}}\right)$ maps are provided in Supplementary Fig. 6a,b, respectively.

Although the number of graphene layers may be determined from the full-width half-maximum (FWHM) of the 2D-band ${ }^{20}$, the absolute value of the FWHM can be affected by the underlying substrate ${ }^{21}$. To avoid such complications, Raman 

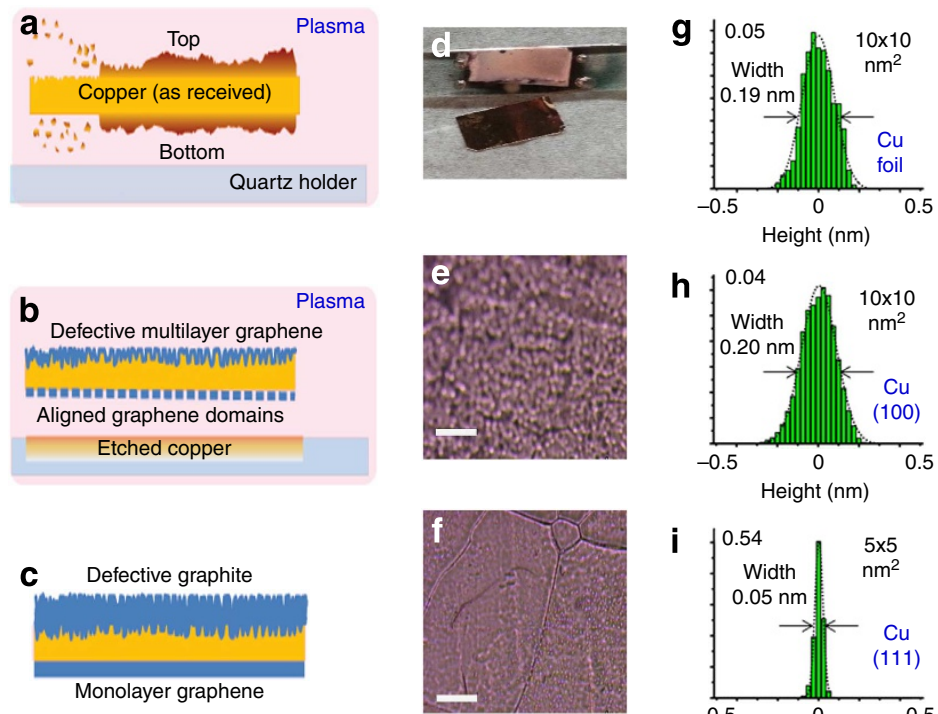
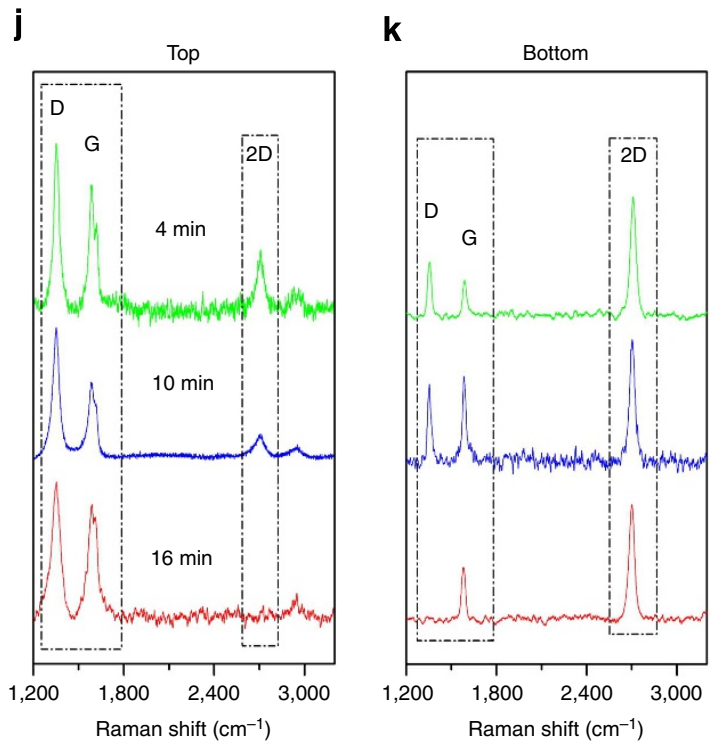

Figure 1 | PECVD-graphene growth process. (a) Exposure to plasma removes the native oxide and smoothes the copper substrate. (b) Aligned graphene nucleates on the bottom of the copper and disordered multilayer graphene forms on the top. (c) Monolayer graphene and disordered carbon develop on the bottom and top of the copper substrate, respectively, with continued exposure to plasma. (d) A copper foil and the sample holder, showing etched copper after PECVD growth. Optical images of the top (e) and bottom (f) of a copper foil after growth, where the scale bars correspond to $50 \mu \mathrm{m}$.

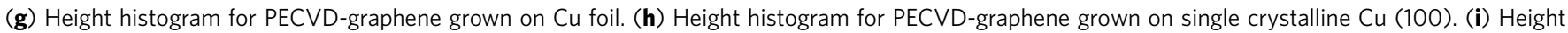
histogram for PECVD-graphene grown on $\mathrm{Cu}(111)$. (j,k) Comparison of the time-evolved Raman spectra of the (j) top and (k) bottom of the Cu foil taken with increasing growth time.

maps of PECVD-graphene transferred from $\mathrm{Cu}$ to $\mathrm{Si} / \mathrm{SiO}_{2}$ are provided in Supplementary Fig. 7a-d and detailed in Methods and Supplementary Note 2. The average value of the FWHM of the $2 \mathrm{D}$-band, which was fit to a single Lorentzian, was $28.8 \mathrm{~cm}^{-1}$, and the average ratio $\left(I_{2 \mathrm{D}} / I_{\mathrm{G}}\right)=2.7$, both consistent with the figure of merit for monolayer graphene ${ }^{15-18,20}$.

High-resolution $(\sim 1 \mathrm{~nm})$ atomic force microscopy and SEM studies over multiple $(100 \times 100) \mu^{2}$ areas of an annealed monolayer graphene sample ${ }^{22}$, which was transferred to single crystalline sapphire with a polymer-free technique ${ }^{23}$, indicated no discernible grain boundaries. With an increase of growth time, small adlayers began to develop on top of the first layer in an aligned manner, as exemplified by the SEM images in Fig. $2 \mathrm{~h}-\mathrm{j}$. These findings, which are supported by Fig. 1k, indicate that the growth of PECVD-graphene on copper foils proceeded by nucleation and growth of well-aligned domains ${ }^{24,25}$ that eventually coalesced into a large single crystalline sheet, and the subsequent growth of the second layer with increasing time followed a similar mechanism (Fig. $2 \mathrm{~h}-\mathrm{j}$ ).

Strain and structural ordering. The biaxial strain $\left(\varepsilon_{11}+\varepsilon_{\mathrm{tt}}\right)$ in the PECVD-graphene on $\mathrm{Cu}$ can be estimated by considering the Raman frequency shifts $\Delta \omega_{\mathrm{m}} \equiv\left(\omega_{\mathrm{m}}-\omega_{\mathrm{m}}^{0}\right)$ and the Gröneisen parameter $\gamma_{\mathrm{m}}^{\text {biax } 15,26 \text { : }}$

$$
\gamma_{\mathrm{m}}^{\mathrm{biax}}=\frac{\Delta \omega_{\mathrm{m}}}{\omega_{\mathrm{m}}^{0}\left(\varepsilon_{\mathrm{ll}}+\varepsilon_{\mathrm{tt}}\right)},
$$

where $\mathrm{m}(=\mathrm{G}, 2 \mathrm{D})$ refers to the specific Raman mode. Using the parameter $\gamma_{2 \mathrm{D}}^{\text {biax }}=2.7$, the strain distribution over an area of $(100 \times 100) \mu \mathrm{m}^{2}$ is exemplified in Fig. $2 \mathrm{~g}$, showing consistently low strain characteristics, with an average strain $\sim 0.07 \%$ for $\mathrm{m}=2 \mathrm{D}$.

Overall, a general trend of downshifted G-band and 2D-band ${ }^{15}$ was found for all PECVD-graphene relative to thermal CVD-grown graphene on the same substrate, as exemplified in
Supplementary Fig. 8a-c for comparison of PECVD- and asgrown thermal CVD-graphene on substrates of $\mathrm{Cu}$ foil, $\mathrm{Cu}$ (100) and $\mathrm{Cu}$ (111). The consistent frequency downshifts for all PECVD-graphene indicate reduction in the averaged biaxial strain. Further, the absence of the D-band in most spectra suggests that the samples were largely free of disorder/edges on the macroscopic scale, which is further corroborated by detailed spatial mapping of the Raman spectra over an area of $(100 \times 100) \mu \mathrm{m}^{2}$ in Fig. $2 \mathrm{e}, \mathrm{f}$.

The PECVD-graphene exhibited a well-ordered honeycomb atomic lattice, which is unique to monolayer graphene, as evidenced by the STM images of samples on $\mathrm{Cu}$ foil (Fig. 3a-c), $\mathrm{Cu}$ (100) (Fig. 3e-g) and $\mathrm{Cu}$ (111) (Fig. 3i-k). The long-range structural ordering was further corroborated by the sharp Fourier transformation (FT) of STM topographies, as exemplified in Fig. 3d,h,l. The FT spectra demonstrated dominantly hexagonal lattices for all PECVD-graphene. Samples grown on $\mathrm{Cu}$ single crystals further exhibited Moiré patterns, as manifested by the second set of smaller Bragg diffraction peaks. Simulations (see Methods) indicated the Moire pattern (a parallelogram) in the FT of Fig. $3 \mathrm{~h}$ for graphene on $\mathrm{Cu}(100)$, was the result of a square lattice at approximately an angle $\sim(12 \pm 2)^{\circ}$ relative to the honeycomb lattice (Supplementary Fig. 9a), whereas the Moiré pattern (a smaller hexagon) in the FT of Fig. 3l suggested that the $\mathrm{Cu}$ (111) hexagonal lattice was at $\sim 6 \pm 2^{\circ}$ relative to the graphene lattice (Supplementary Fig. 9b). These STM measurement over multiple samples and multiple areas per sample confirm the predominance of monolayer graphene and the absence of discernible bilayer graphene in our PECVD-grown samples.

The STM topography was further employed to analyse strain at the microscopic scale. For a local two-dimensional displacement field $\mathbf{u}=u_{x} \hat{x}+u_{y} \hat{y} \equiv \mathbf{r}-\mathbf{r}_{0}$, where $\mathbf{r}$ and $\mathbf{r}_{0}$ denote the actual position of a carbon atom and its equilibrium position in ideal graphene, respectively ${ }^{27}$, the compression/dilation strain is given by $\left(\partial u_{x} / \partial x\right)+\left(\partial u_{y} / \partial y\right) \equiv u_{x x}+u_{y y}$, which is proportional to the biaxial strain ${ }^{5,7,8}$. Using the topographies shown in Fig. $3 a-c$, we 

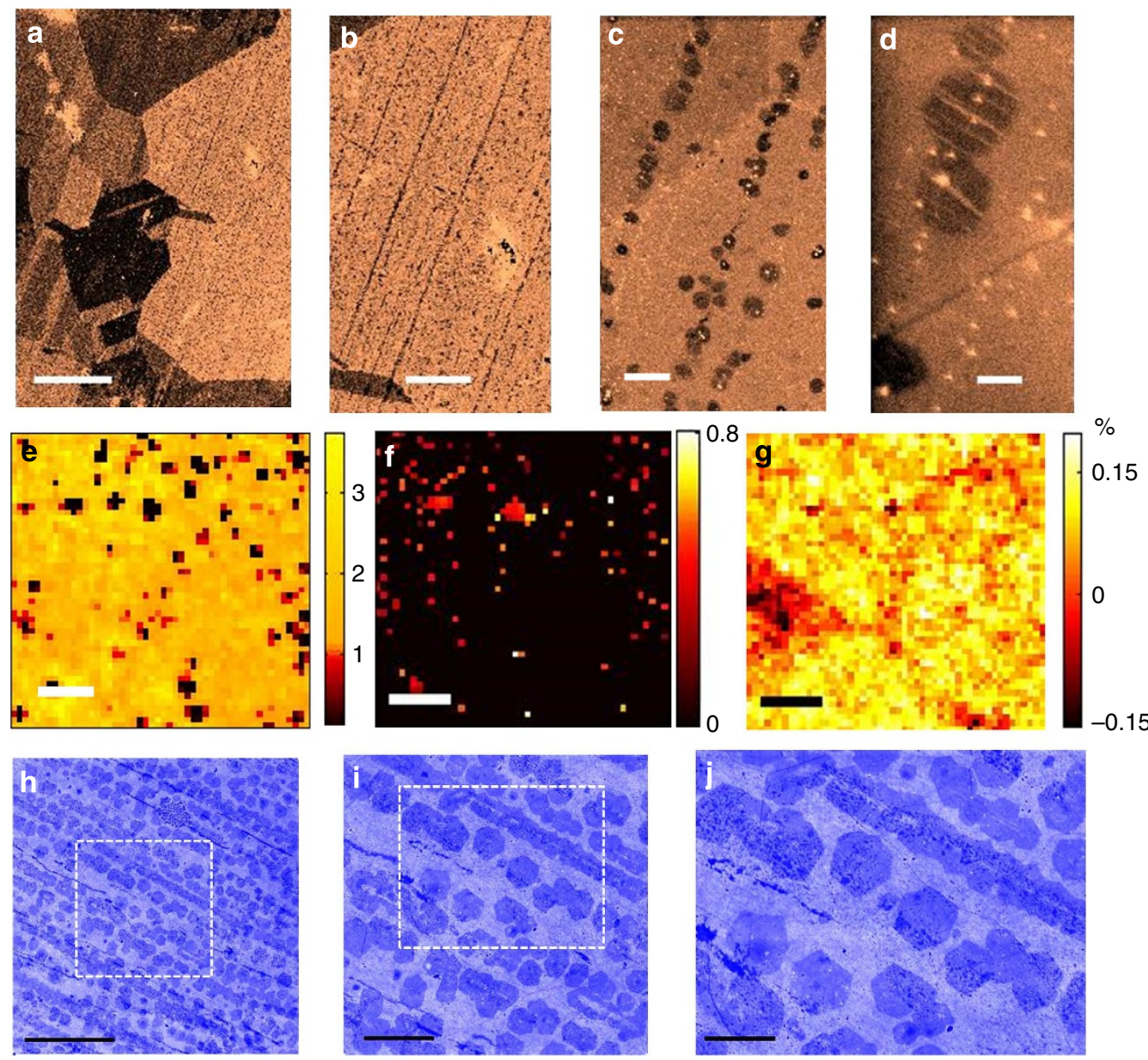

Figure 2 | Large-area characterization of PECVD-graphene on Cu. (a-d) False-colour SEM images of early-stage growth (with increasing magnification from left to right, where the scale bars correspond to $30 \mu \mathrm{m}, 10 \mu \mathrm{m}, 1 \mu \mathrm{m}$ and $200 \mathrm{~nm}$, respectively), showing extended linear arrays of well-aligned hexagonal domains (dark) on copper foil (light). (e) $(100 \times 100) \mu \mathrm{m}^{2}$ map (scale bar, $\left.20 \mu \mathrm{m}\right)$ of the Raman spectral $2 \mathrm{D} / \mathrm{G}$ intensity ratio of a fully developed monolayer graphene sample on copper foil. (f) $(100 \times 100) \mu \mathrm{m}^{2}$ map (scale bar: $20 \mu \mathrm{m}$ ) of the Raman spectral D/G intensity ratio over the same area as in $\mathbf{e} ;(\mathbf{g})(100 \times 100) \mu \mathrm{m}^{2}$ strain map (scale bar, $\left.20 \mu \mathrm{m}\right)$ over the same area as in $\mathbf{e}$ and $\mathbf{f}$. (h-j) False-colour SEM images of graphene grown for excessive time and transferred to single crystalline sapphire (with increasing magnification from left to right, where the scale bars corresponding to 30 , 10 and $5 \mu \mathrm{m}$, respectively), showing well-aligned adlayer graphene domains (dark) on the bottom monolayer graphene (light).

obtained the spatial strain maps for PECVD-graphene on $\mathrm{Cu}$ foil, $\mathrm{Cu}(100)$ and $\mathrm{Cu}(111)$ substrates over successively decreasing areas in Fig. 4a,b,e,f,i,j, respectively. The corresponding strain histograms are given in Fig. 4c,g,k. The PECVD-graphene exhibited low and relatively homogeneous strain distributions. Further comparison with the macroscopic strain obtained from a collection of Raman spectra taken on different areas of multiple PECVD-graphene samples are summarized by the strain histograms in Fig. 4d,h,l. There is overall consistency between microscopic STM studies and macroscopic Raman spectroscopic studies, revealing low strain for all PECVD-graphene.

Electrical properties. The electrical mobility $(\mu)$ of PECVDgraphene was determined by studying back-gated field-effecttransistor devices ${ }^{28,29}$. Graphene samples were first transferred to hexagonal boron nitride (BN) thin films on $300 \mathrm{~nm} \mathrm{SiO}_{2} / \mathrm{Si}$ substrates using a polymer-free method $^{23}$, and then lithographically processed into a geometry shown in Fig. 5a,b. The sheet resistance of graphene as a function of the back-gate voltage $(V)$ and the corresponding conductivity $(\sigma)$ versus sheet carrier density $\left(n_{\mathrm{s}}\right)$ were measured at $300 \mathrm{~K}$, as exemplified in Fig. $5 c$,d. The electrical mobility $\mu$ was obtained from the derivative of the Drude formula ${ }^{28}$ near the charge neutrality point:

$$
\mu=(1 / C) \mathrm{d} \sigma / \mathrm{d} V
$$

where $C$ denotes the capacitance of the device. The electrical mobility obtained from nine different devices was found to range from $(3.0 \pm 0.5) \times 10^{4}$ to $(6.0 \pm 1.0) \times 10^{4} \mathrm{~cm}^{2} \mathrm{~V}^{-1} \mathrm{~s}^{-1}$ for electrons, as summarized in Table 1 , and from $(1.2 \pm 0.2) \times 10^{4}$ to $(3.5 \pm 0.5) \times 10^{4} \mathrm{~cm}^{2} \mathrm{~V}^{-1} \mathrm{~s}^{-1}$ for holes, where the errors represented variations in determining the $(\mathrm{d} \sigma / \mathrm{d} V)$ slope due to the discreteness of voltage readings. These values are comparable to those obtained on large, single crystalline thermal CVD-grown graphene on $\mathrm{BN}$, the latter yielded $\mu=4 \times 10^{4}$ to $6 \times$ $10^{4} \mathrm{~cm}^{2} \mathrm{~V}^{-1} \mathrm{~s}^{-1}$ at $1.7 \mathrm{~K}$ and $\sim 1.5 \times 10^{4}$ to $3 \times 10^{4} \mathrm{~cm}^{2} \mathrm{~V}^{-1}$ $\mathrm{s}^{-1}$ at $300 \mathrm{~K}$ (ref. 2).

It is worth commenting on the accuracy of mobility values obtained by the two-terminal configuration depicted in Fig. 5a,b. The contact resistance between electrode and graphene was determined by the Transmission Line Model method and was found to be typically $\sim 10 \Omega$ for all devices. In contrast, the sheet resistance of graphene was typically more than $1 \mathrm{k} \Omega$ near the charge neutrality point, which was about two orders of magnitude larger than the contact resistance. Therefore, the contact 

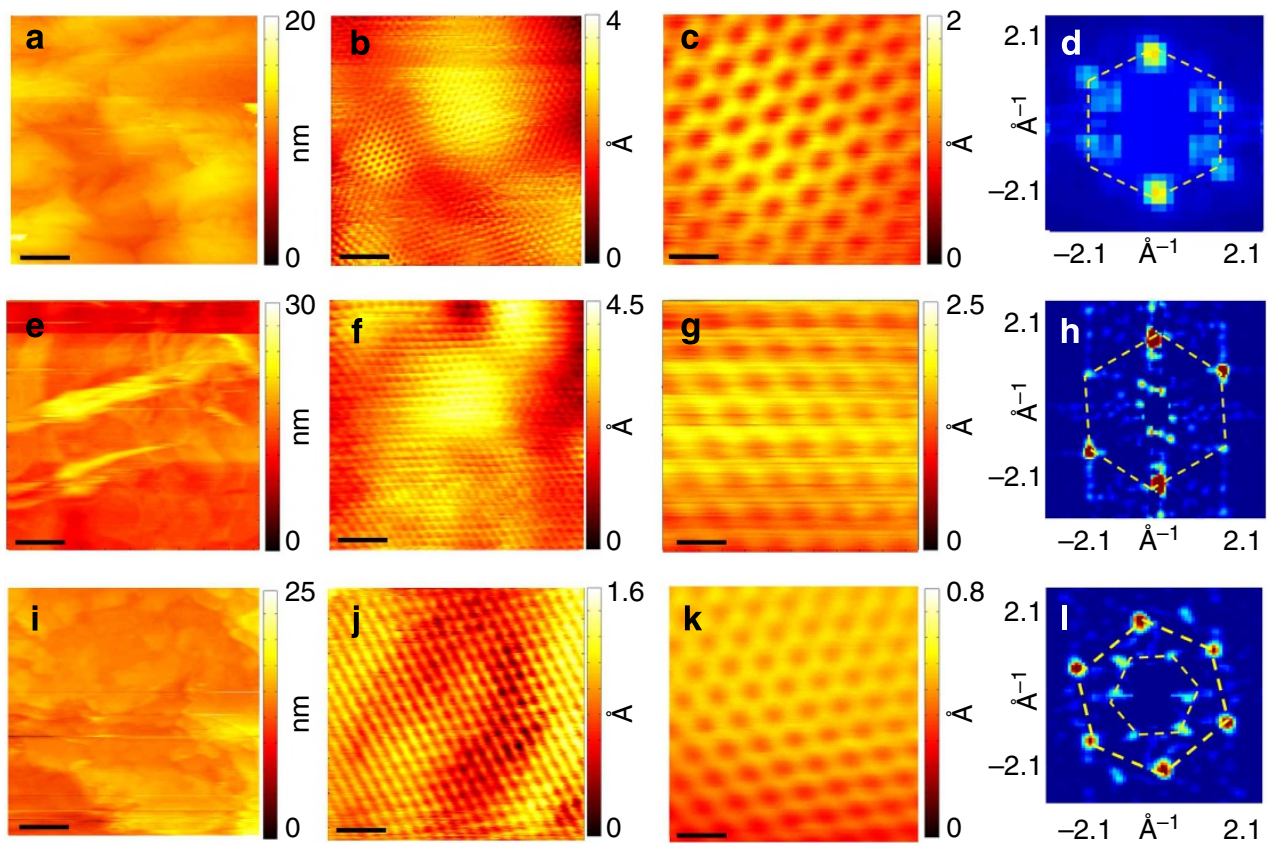

Figure 3 | Topographies of PECVD-graphene on copper. STM topographies of PECVD graphene at 77 K over successively decreasing areas (first three columns) and the corresponding Fourier transformation (FT) of large-area topography (fourth column) for samples grown on (a-d) Cu foil; (e-h) Cu (100); and (i-I) $\mathrm{Cu}$ (111). The scale bars for $\mathbf{a}, \mathbf{e}$ and $\mathbf{i}$ are $40 \mathrm{~nm}$; for $\mathbf{b}, \mathbf{f}$ and $\mathbf{j}$ are $2 \mathrm{~nm}$; and for $\mathbf{c}, \mathbf{g}$ and $\mathbf{k}$ are $0.4 \mathrm{~nm}$.
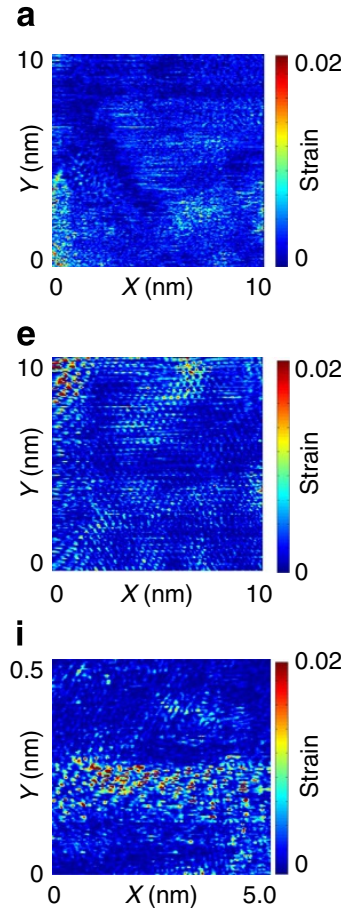

b

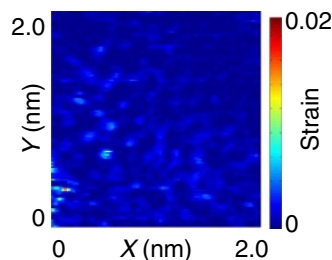

$$
\text { f }
$$

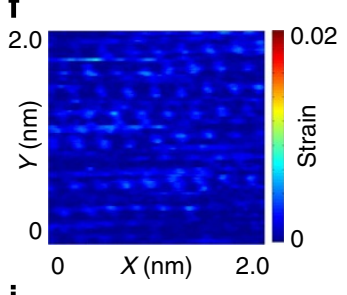

j

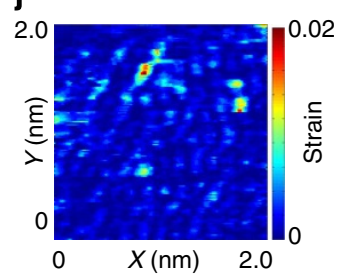

C

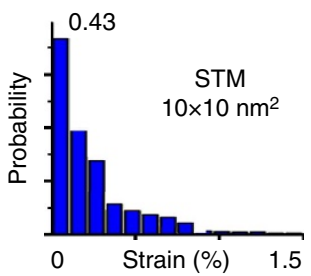

g

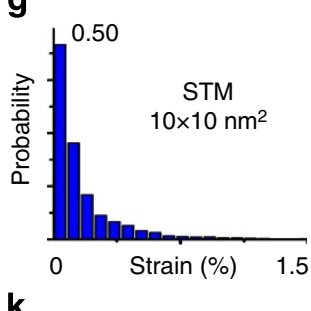

k

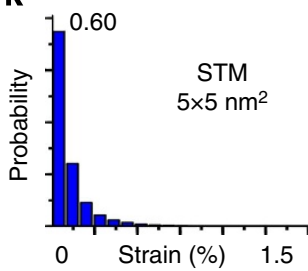

d

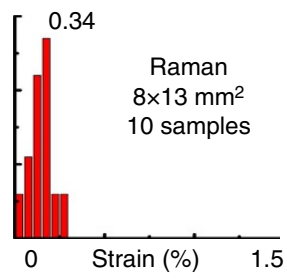

h

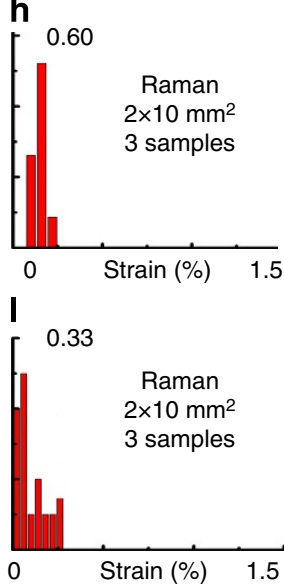

Figure 4 | Comparison of the spatially resolved strain maps and strain histograms of PECVD-graphene. From left to right, compression/dilation strain maps over successively decreasing areas taken with STM at $77 \mathrm{~K}$ (first and second columns, colour scale in units of \%), strain histogram (third column) of the strain map shown in the first column, and strain histogram (fourth column) obtained from Raman spectroscopic studies of different areas of multiple PECVD-graphene samples grown on (a-d) Cu foil, (e-h) Cu (100) and (i-I) Cu (111). The strain obtained from STM topography is largely consistent with the findings from Raman spectroscopic studies.

resistance would not affect the accuracy of the mobility significantly. Additional measurements by patterning some of the PECVD-graphene into the four-point configuration revealed that the differences in mobility thus determined was less than 2-3\% from those obtained by means of the two-point method.

\section{Discussion}

Experience with diamond PECVD lends understanding to the observed nucleation and growth of aligned graphene domains presented here. In the case of diamond growth, it is known to involve a competition between growth by carbon radicals (most 
a

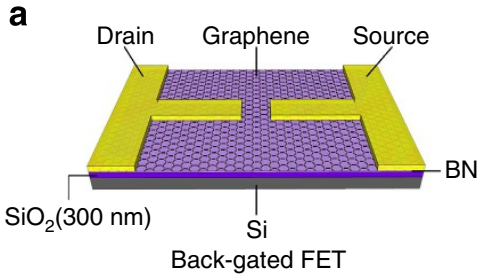

C

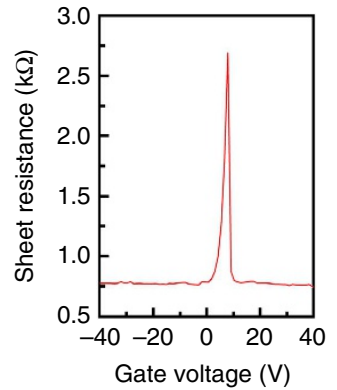

b

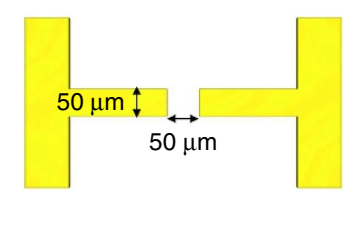

d

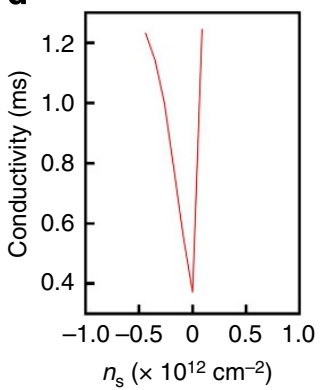

Table 1 | Electron mobility $(\mu)$ data from nine different FET devices based on PECVD-graphene.

\begin{tabular}{|c|c|}
\hline Device & Electron mobility $\left(\mathrm{cm}^{2} \mathrm{v}^{-1} \mathrm{~s}^{-1}\right)$ \\
\hline 1 & 54,000 \\
\hline 2 & 52,000 \\
\hline 3 & 42,000 \\
\hline 4 & 38,000 \\
\hline 5 & 31,000 \\
\hline 6 & 30,000 \\
\hline 7 & 55,000 \\
\hline 8 & 60,000 \\
\hline 9 & 38,000 \\
\hline Average & 44,400 \\
\hline
\end{tabular}

system (Opthos Instruments Inc.) consists of an Evenson cavity and a power supply (MPG-4), which provides an exciting frequency of 2,450 MHz. The Evenson cavity mates with a quartz tube of inner and outer diameters of 10 and $12.5 \mathrm{~mm}$, respectively. The vacuum system is comprised of a mechanical roughing pump, two capacitance manometers, a pressure control valve and a measurement control system. There is a fore-line trap between the vacuum pump and the MKS 153 control valve. The gas delivery system consists of mass flow controllers (MFCs) for $\mathrm{H}_{2}, \mathrm{CH}_{4}$ and Ar. A bakeable variable leak valve is placed before the methane MFC, and there is a leak valve for $\mathrm{N}_{2}$. A gas purifier was placed before the variable leak valve on the methane line. Quarter-turn, shut-off valves are placed directly after each of the MFCs. The system pressure and gas flows are monitored and controlled through the controller via a LabView interface. A residual gas analyzer (RGA) is used to monitor the exhaust gas.

Detailed PECVD growth procedure and information. The copper substrates were placed on a quartz flat inside of quartz tube. A typical substrate size was $(8 \times 13) \mathrm{mm}^{2}$. The tube was evacuated to $25-30$ mTorr. A 2-5 standard-cubiccentimeters-per-minute (s.c.c.m.) flow of room temperature hydrogen gas with $0.4 \%$ methane and a comparable amount of nitrogen gas was added and the pressure was controlled at $500 \mathrm{mTorr}$.

The addition of methane to the gas flow was controlled by a precision leak valve, and a typical concentration, as measured by gas chromatography, was $0.4 \%$. The initially low vacuum conditions ( $\sim 25$ mTorr $)$ provided sufficient partial pressures of nitrogen (atmospheric) for the PECVD process. (We note that a leak valve for including purified nitrogen was added to the setup to study the effects of increased nitrogen partial pressures.) Typical partial pressures measured by RGA are as follows:

$\mathrm{N}_{2}$ (Mass 28): $6.9 \times 10^{-8}$ Torr

$\mathrm{CH}_{4}$ (Mass 16): $1.5 \times 10^{-7}$ Torr

$\mathrm{O}_{2}$ (Mass 32): $5.4 \times 10^{-9}$ Torr

$\mathrm{H}_{2} \mathrm{O}$ (Mass 18): $1.2 \times 10^{-6}$ Torr

$\mathrm{CO}_{2}$ (Mass 44): $2.3 \times 10^{-8}$ Torr

From these values, we estimate that the concentration of nitrogen in the gas flow is typically on the same order as that of methane.

The PECVD process was found to be highly sensitive to the relative amounts of methane and nitrogen. Excessive methane mixtures resulted in no etching, whereas excessive nitrogen mixtures would result in excessive etching of the copper. Slight adjustments to the methane concentration could be made via either optical emission spectroscopy or RGA.

A low-power (ranging from 10 to $40 \mathrm{~W}$ ) cold hydrogen plasma was formed over the copper substrate using an Evenson cavity. Exposure of copper substrates to the plasma enabled continuing etching and cleansing of the copper surface during the graphene growth process. The gas temperature inside the plasma was measured using a thermocouple sheathed in boron nitride. The thermocouple was placed in the plasma above the sample and could be translated along the tube. The peak gas temperature measured in plasma treatment was $160^{\circ} \mathrm{C}$ at $10 \mathrm{~W}$ and $425^{\circ} \mathrm{C}$ at $40 \mathrm{~W}$, and the gas temperature profile decreased rapidly (by $120^{\circ} \mathrm{C}$ at $10 \mathrm{~W}$ and $425^{\circ} \mathrm{C}$ at $40 \mathrm{~W}$ within $1 \mathrm{~cm}$ ) from the peak value. The maximum temperature of the copper substrate $T_{\mathrm{s}}$ was measured using the melting point of known solids, lead and zinc, and found to be within the range of $327.5^{\circ} \mathrm{C}<T_{\mathrm{s}}<419.5^{\circ} \mathrm{C}$ at $40 \mathrm{~W}$. Typically after 5-20 min of direct exposure to the plasma, a large-area monolayer graphene formed on the backside of copper substrates, whereas the front side of the substrates was coated with disordered graphite. Upon the completion of graphene growth, the plasma was extinguished, and the gas flows were stopped. The process tube was then evacuated and back filled with Ar, and the substrate with graphene coating was subsequently removed. Copper deposition was visible on the inside of the tube and on the sample holder as the result of plasma etching of the copper substrates.
Experimental setup. The experimental setup is summarized in Supplementary Fig. 1, which consists of plasma, vacuum and gas delivery systems. The plasma 
Analysis of monolayer graphene using Raman spectroscopy. The Raman maps shown in Fig. 2e,f of the manuscript for PECVD-graphene on $\mathrm{Cu}$ were collected with Renishaw InVia @ $532 \mathrm{~nm}$, and the spectra were taken at $2 \mu \mathrm{m}$ per pixel steps over an area of $(100 \times 100) \mu \mathrm{m}^{2}$ for a total of 2,601 spectra. Similarly, the Raman maps in Supplementary Fig. 7a,b for PECVD-graphene transferred to $\mathrm{SiO}_{2}$ were taken at $2 \mu \mathrm{m}$ per pixel steps over an area of $(160 \times 150) \mu \mathrm{m}^{2}$. Each spectrum was smoothed and the broad background from the copper fluorescence was removed, and the peak locations and intensities for the $\mathrm{D}, \mathrm{G}$ and $2 \mathrm{D}$ features were extracted. The relative intensities of the $2 \mathrm{D}$ and G-band and those of the D and G-band are presented in Fig. 2e,f, and histograms of the ratio values and the FWHM linewidth of the 2D band of our PECVD-grown graphene on $\mathrm{Cu}$ and commercial thermal $\mathrm{CVD}$-grown graphene on $\mathrm{Cu}$ are shown in Supplementary Fig. 6a-e. The predominant $\left(I_{\mathrm{D}} / I_{\mathrm{G}}\right) \sim 0$ value of the graphene sample shown in Supplementary Fig. 6a indicates negligible defects, whereas the histogram of the $2 \mathrm{D} / \mathrm{G}$ intensity ratio $\left(I_{2 \mathrm{D}} /\right.$ $\left.I_{\mathrm{G}}\right)$ shown in Supplementary Fig. 6b reveals that the average of the $\left(I_{2 \mathrm{D}} / I_{\mathrm{G}}\right)$ value is $\sim 2$, which agrees with the figure of merit $\left(I_{2 \mathrm{D}} / I_{\mathrm{G}}\right)>1$ for monolayer graphene ${ }^{15-18}$.

This notion of predominantly monolayer graphene described in this work is further corroborated by detailed studies of a PECVD-graphene sample transferred from $\mathrm{Cu}$ to a $\mathrm{SiO}_{2}$ substrate, which prevents the complication of slow oxidation of the $\mathrm{Cu}$ substrate that could result in the $2 \mathrm{D}$ linewidth broadening with time due to the influence of the substrate (see Supplementary Note 2). As shown in Supplementary Fig. $7 \mathrm{a}-\mathrm{d}$, a large spatial map over an area of $(160 \times 150) \mu \mathrm{m}^{2}$ revealed a mean FWHM value $=28.8 \mathrm{~cm}^{-1}$ for the $2 \mathrm{D}$-band and a mean value $\left(I_{2 \mathrm{D}} / I_{\mathrm{G}}\right)=2.7$, both are consistent with predominantly monolayer graphene if we use either the criterion of 2D-band FWHM $<30 \mathrm{~cm}^{-1}$ as the figure of merit for monolayer graphene on $\mathrm{SiO}_{2}$ (ref. 20) or the criterion $\left(I_{2 \mathrm{D}} / I_{\mathrm{G}}\right)>1$ for monolayer graphene on various substrates ${ }^{15-18}$.

Simulations. Simulations of the Moire patterns and the corresponding FT were made by means of Matlab. We generated the triangular and cube lattices by using cosine square function and the hexagonal lattice by displacement of two triangular lattice functions. The lattice constant for the ideal $2 \mathrm{D}$ honeycomb structure of graphene is $0.2461 \mathrm{~nm}$ and that for the ideal Cu FCC lattice is $0.3615 \mathrm{~nm}$, which has a corresponding lattice constant $(0.3615 / \sqrt{2})=0.2556 \mathrm{~nm}$ for the triangular lattice in the 111 direction and the square lattice in the 100 direction.

To simulate realistic STM imaging of the Moire pattern for a given relative angle $\theta$ between the top graphene layer and the underlying $\mathrm{Cu}$ lattice, we assume the resulted topological function $G(x, y)$ has the following form:

$$
G(x, y)=G_{\mathrm{C}}(x, y)+r e^{-k G_{\mathrm{C}}(x, y)} G_{\mathrm{Cu}}(x, y, \theta),
$$

where $0<r \leq 1, G_{\mathrm{C}}(x, y)$ is the graphene honeycomb lattice function and $G_{\mathrm{Cu}}(x, y, \theta)$ is the copper layer function which could be either the triangular lattice for $\mathrm{Cu}$ (111) or the cubic lattice for $\mathrm{Cu}(100)$. Both functions are normalized with maximum 1 and minimum 0 . We use the exponent $e^{-k G_{C}(x, y)}$ in equation (3) to simulate the rapid decay of the tunnelling current contribution from the copper atom directly under a carbon atom in the top graphene layer, where $k$ is a parameter that controls the decay rate and was chosen to be 2-4. Even for exposed copper atoms without any carbon atom directly positioning above, the signal from copper is expected to be attenuated by a ratio $r$ due to a larger distance from the STM tip to the copper layer. The above expression ensures that contributions from copper atoms directly under carbon atoms are much reduced due to deflection of tunnelling electrons, whereas those from exposed copper atoms are only attenuated by a coefficient $r$.

The matching between simulations and the Fourier transform (FT) of graphene on $\mathrm{Cu}(100)$ lattice data is judged by the angle of inner parallelism. At $\theta=(12 \pm 2)^{\circ}$, the angle of the inner parallelism appear to match the experimental result in Fig. $3 \mathrm{~h}$, as shown in by the real space Moire pattern (left panel) and the corresponding FT (right panel) in Supplementary Fig. 9a.

In contrast, the matching between simulations and the FT of graphene on $\mathrm{Cu}$ (111) data is based on the size of inner hexagon relative to that of the outer hexagon for the graphene lattice. In fact, there are actually two inner hexagons according to the simulations, as exemplified in the FT of the Moire pattern in Supplementary Fig. $9 \mathrm{~b}$ for $\theta=(6 \pm 2)^{\circ}$. However, empirically only the larger inner hexagon is visible (Fig. 3l). We attribute this discrepancy to the intense DC signal from the zone centre of the FT spectra, which overwhelms the signal of the smaller inner hexagon and so becomes invisible upon our removal of the DC signal.

\section{References}

1. Li, X. et al. Large-area synthesis of high-quality and uniform graphene films on copper foils. Science 324, 1312 (2009).

2. Hao, Y. et al. The role of surface oxygen in the growth of large single-crystal graphene on copper. Science 342, 720 (2013).

3. Yan, Z. et al. Toward the synthesis of wafer-scale single-crystal graphene on copper foils. ACS Nano 6, 9110 (2012).

4. Mun, J. H. \& Cho, B. J. Synthesis of monolayer graphene having a negligible amount of wrinkles by stress relaxation. Nano Lett. 13, 2496 (2013).

5. Guinea, F., Katsnelson, M. I. \& Geim, A. K. Energy gaps and a zero-field quantum Hall effect in graphene by strain engineering. Nat. Phys. 6, 30 (2010).

6. Levy, N. et al. Strain-induced pseudo magnetic fields greater than 300 Tesla in graphene nanobubbles. Science 329, 544 (2010).
7. Yeh, N.-C. et al. Strain-induced pseudo-magnetic fields and charging effects in CVD-grown graphene. Surf. Sci. 605, 1649 (2011).

8. Yeh, N.-C. et al. Scanning tunneling spectroscopic studies of Dirac fermions in graphene and topological insulators. EPJ Web. Conf. 23, 00021 (2012).

9. Goodwin, D. G. \& Butler, J. E. Handbook of Industrial Diamonds and Diamond Films (Marcel Dekker, Inc., 1997).

10. Kalita, G. et al. Low temperature growth of graphene film by microwave assisted surface wave plasma CVD for transparent electrode application. RSC Adv 2, 2815 (2012).

11. Yamada, T., Ishihara, M. \& Hasegawa, M. Large area coating of graphene at low temperature using a roll-to-roll microwave plasma chemical vapor deposition. Thin Solid Films 532, 89 (2013).

12. Nandamuri, G., Roumimov, S. \& Solanki, R. Remote plasma assisted growth of graphene films. Appl. Phys. Lett. 96, 154101 (2010).

13. Kuboi, N. \& Fukusawa, M. Plasma treatment method, plasma treatment apparatus, and semiconductor device manufacturing method. US patent 20130017672 Al (2013).

14. Shard, A. G. Detection limits in XPS for more than 6000 binary systems using $\mathrm{Al}$ and $\mathrm{Mg} \mathrm{K} \alpha$ X-rays. Surf. Interface Anal. 46, 175 (2014).

15. Ferrari, A. C. et al. Raman spectrum of graphene and graphene layers. Phys. Rev. Lett. 97, 187401 (2006).

16. Wu, W. et al. Control of thickness uniformity and grain size in graphene films for transparent conductive electrodes. Nanotechnology 23, 035603 (2012).

17. Dimiev, A. et al. Layer-by-layer removal of graphene for device patterning. Science 331, 1168 (2011)

18. Zhou, H. et al. Chemical vapour deposition growth of large single crystals of monolayer and bilayer graphene. Nat. Commun. 4, 2096 Article number: 3096.

19. Celebi, K. et al. Observations of early stage graphene growth on copper. Electrochem. Solid-State Lett 15, K1 (2012).

20. Lee, S. et al. Wafer scale homogeneous bilayer graphene films by chemical vapor deposition. Nano Lett. 10, 4702 (2010).

21. Yin, X. et al. Evolution of the Raman spectrum of graphene grown on copper upon oxidation of the substrate. Nano Res. 1-10 (2014) doi:10.1007/ s12274-014-0521-0.

22. Nemes-Incze, P. et al. Revealing the grain structure of graphene grown by chemical vapor deposition. Appl. Phys. Lett. 99, 023104 (2011).

23. Lin, W.-H. et al. A direct and polymer-free method for transferring graphene grown by chemical vapor deposition to any substrate. ACS Nano 8, 1784-1791 (2014).

24. Murdock, A. T. et al. Controlling the orientation, edge geometry, and thickness of chemical vapor deposition graphene. ACS Nano 7, 1351 (2013).

25. Lee, J.-H. et al. Wafer-scale growth of single-crystal monolayer graphene on reusable hydrogen-terminated germanium. Science 344, 286 (2014).

26. Ferralis, N., Maboudian, R. \& Carraro, C. Evidence of structural strain in epitaxial graphene layers on $6 \mathrm{H}-\mathrm{SiC}(0001)$. Phys. Rev. Lett. 101, 156801 (2008).

27. Teague, M. L. et al. Evidence for strain-induced local conductance modulations in single-layer graphene on $\mathrm{SiO}_{2}$. Nano Lett. 9, 2542 (2009).

28. Gannett, W. et al. Boron nitride substrates for high mobility chemical vapor deposited graphene. Appl. Phys. Lett. 98, 242105 (2011).

29. Dean, C. R. et al. Boron nitride substrates for high-quality graphene electronics. Nat. Nanotechnol 5, 722 (2010).

30. Mankelevich, Yu. A. \& May, P. W. New insights into the mechanism of CVD diamond growth: Single crystal diamond in MW PECVD reactors. Diam. Relat. Mater. 17, 1021 (2008).

31. Liu, H. \& Dandy, D. S. Diamond Chemical Vapor Deposition: Nucleation and Early Growth Stages (Elsevier, 1996).

32. Jiang, N. et al. Interfacial analysis of CVD diamond on copper substrates. Diam. Relat. Mater 6, 743 (1997).

\section{Acknowledgements}

This work at Caltech was supported by National Science Foundation under the Institute of Quantum Information and Matter, and by Moore and Kavli Foundations through the Kavli Nanoscience Institute. The work in Taiwan was supported by the National Science Council under contracts 100-2911-I-002-514 and 101-2628-M-002-004. We thank Professor George Rossman for the use of his Raman spectrometer. D.A.B. specially thanks late Professor David G. Goodwin for his friendship and mentorship.

\section{Author contributions}

D.A.B. conceived the PECVD-graphene growth idea. D.A.B., W.-H.L. and C.-C.H. developed the PECVD-graphene growth procedures and carried out Raman spectroscopic characterizations. C.-C.H. carried out SEM and atomic force microscopy studies on graphene samples. W.-H.L. processed the graphene FET devices and carried out the mobility measurements and analysis. Y.-Y.L, W.-B.S., C.-S.C. and M.L.T. performed the STM studies. T.-C. C. performed the XPS studies and analysis of the graphene samples. C.-I. Wu coordinated the device fabrication, mobility, XPS and part of the STM experiments. C.-C.C. and M.L.T. conducted the simulations and analysis of the STM 
data. N.-C.Y. coordinated with all co-authors on the design, planning and execution of the experiments, data analysis and simulations, and wrote the manuscript together with D.A.B.

\section{Additional information}

Supplementary Information accompanies this paper at http://www.nature.com/ naturecommunications
Competing financial interests: The authors declare no competing financial interests.

Reprints and permission information is available online at http://npg.nature.com/ reprintsandpermissions/

How to cite this article: Boyd, D. A. et al. Single-step deposition of high-mobility graphene at reduced temperatures. Nat. Commun. 6:6620 doi: 10.1038/ncomms7620 (2015). 